\title{
Modelling of IR images of sky and clouds
}

by T. Sosnowski*, R. Dulski* and H. Polakowski*

* Military University of Technology, Institute of Optoelectronics, ul. Gen. Sylwestra Kaliskiego 2, 00-908 Warsaw, Poland

\section{Abstract}

A phenomenon of thermal radiation of real objects is used in systems of automatic target recognition (ATR). To develop effective algorithms, it is essential to know both radiation characteristics of the detected objects and characteristics of their surroundings. The paper presents a method of numerical modeling of clouds radiation and their environment in IR range. Experimental data registered with short wavelength and long wavelength IR cameras were used. The experimental data were collected for characteristic temperature profiles of clouds and sky, for particular seasons of the year and metrological conditions occurring in Poland. To generate virtual images of sky and clouds in IR, IR Sky software has been developed. The images generated by means of this software were analyzed, having in view influence of radiation features of sky and clouds on a thermodetection process. High consistency of computer-generated images with the real ones (registered) thermal imagess has been obtained.

\section{Introduction}

In detection and recognition of objects it is important to have high number of data sets for object and various variants of background on which the object is situated. Adequately high number of data variants allows for development of efficient algorithms of detection and recognition of objects, independently of a background. Simultaneously, the variants of object parameters and background can be used in a process of teaching, e.g., artificial neuron networks. In all cases, indispensable is to have a lot of background examples. The most often it is not possible to register enough number of patterns (object and background) allowing for development and verification of an algorithm or efficient teaching of the device. Moreover, it is difficult to register all possible combinations of parameters of an object and a background, e.g., showing full scale of atmospheric changes. Having in view the above mentioned reasons, very often an object and a background are modeled [5, 6, 8, 12, 13, $14,15]$, what provides generation of adequate number of data variants.

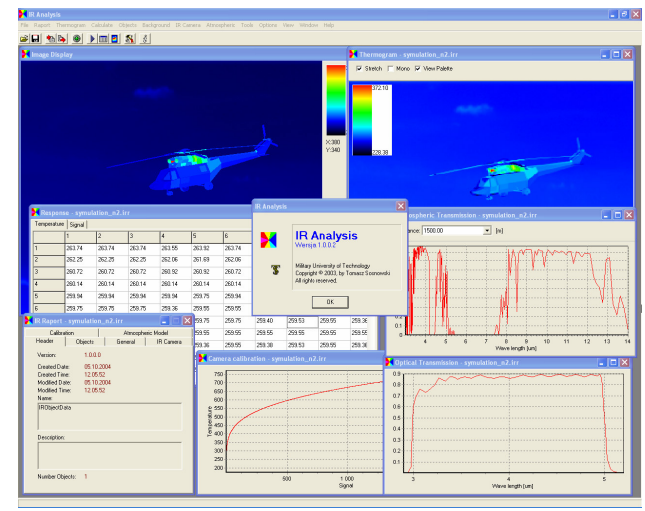

Fig. 1. A window of IR analysis program

An example of a system for simulation and analysis of a thermal scene in 3D is the designed and performed, at the Military University of Technology, special software IR Analysis [5, 6] (Fig. 1). This software can be used for simulation and analysis of a thermal scene in 3D on the basis of geometrical parameters and thermal properties of the being observed object, parameters of virtual imaging device, and environment parameters (e.g., spectral transmission of the atmosphere). The software generates a thermal image for the determined observation conditions, so-called, measuring situation.

Main elements of the system for simulation and analysis of a thermal scene are: virtual imaging device (camera), virtual thermal object, and a background model. The system, on the basis of the defined location of an object, background parameters, environment parameters (e.g., atmosphere transmission) and geometrical representation of scene elements on a plane of a detectors array (matrix) determines the signals (responses) of detectors $[6,8,10,12,15]$. Next, using the calibration data (calibration characteristic), temperature is given.

The value of an analogue signal is influenced mainly by a camera and an observed object as well as a background, environment and transmission channel. The camera is an optoelectronic device converting electromagnetic radiation into electric signal $[10,12,13,15]$. In general, the detector can consist of a lot of active elements. Radiation, reaching the detector, passes earlier through the objective or through an additional optical 
filter. Moreover, the detector is closed with the input window. All these elements affect both intensity and spectral distribution of radiation that passes through them.

Radiation, reaching the camera's detector, is emitted by the object, background, and environment. It is modified in a transmission channel, in which its intensity and spectral distribution are changed. The whole modeling process takes place in $3 \mathrm{D}$, so-called a thermal scene $[5,6,8,12,13]$.

A computer model of a thermal object describes its radiation properties in IR range. The model is devoted to simulation of properties of a real object in investigations of influence of thermal scene conditions on the possibilities of object detection by a thermodetection system. In the software, a model is implemented as a set of plane surfaces. Moreover, the model contains the information on a temperature distribution and an emission coefficient on its surface. The data describing the object allow for determining the radiation emission from the object surface at various observation directions.

Important parameters are the environmental parameters describing thermal properties of the space surrounding a measuring system and determining the conditions of radiation propagation in IR range. These parameters are, among others: atmospheric spectral transmission, ambient temperature, humidity, and the like

Thermal background, registered by thermodetection devices, is significantly diversified. In a thermal background image, natural elements can appear such as elevations, trees, clouds as well as elements of infrastructure created by people, e.g., high buildings, chimneys, towers, masts. All these elements have temperature distributions significantly dependent on atmospheric conditions. For flying objects, frequently, a background forms the sky and clouds.

\section{Sky and clouds model in IR}

A precise meteorological mathematical models are too complicated to use them in a system simulating sky and clouds on it for simulation of thermal camera operation. Harris model [2], limited only to the calculations connected with clouds dynamics is sufficient but only for a short range of about $3 \mathrm{~km}$. From the point of view of an observer (thermal camera) situated on the Earth's surface this range is so small part of the sky that it is not sufficient for thermal scene simulation. In modeling, based on cellular automata, some difficulties connected with selection of logical functions appear. Moreover, the most frequently, they can simulate lonely some kinds of clouds.

Basing on observation of clouds, a list of their features can be created. The most important are:

- clouds are moving in the sky and their shape does not change in time,

- number of clouds in the sky changes continuously

- $\quad$ structures of clouds are similar

- cloud cover changes from overcast to clear sky only with several separate clouds (or without them),

- clouds shape changes in time,

- clouds are 3D objects,

- clouds are illuminated by the Sun,

- clouds are at the same height over the Earth.

Analyzing the thermal imagess of real clouds [7, 9], it can be described that from the point of view of a thermal camera, the following kinds of cloud cover appear: cloudless sky (clear sky), single clouds, cloudy sky, and broken sky.

For cloudless sky, the sky temperature registered by a thermal camera is very low and frequently it is limited by the lower measuring range of a camera. It can be taken for simulation that cloudless sky has the Gaussian temperature distribution of low average value and relatively low variance.

Cloudy sky has a temperature measurable with the camera that depends, among others, on a spectral range of a thermal camera. It can be taken for simulation that cloudy sky has the Gaussian temperature distribution of an average value dependent on a spectral range of the camera and intensity of sun irradiation and of relatively high variance $[7,9]$.

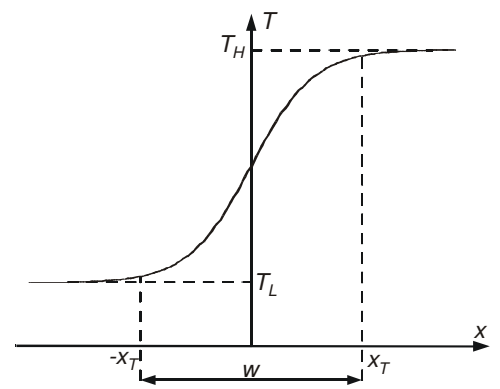

Fig. 2. Characteristic of a function approximating temperature change at a cloud edge

In case of single clouds, the temperature inside the cloud is of random character of normal distribution of a relatively high average value (similarly as for cloudy sky). Out of the cloud, the temperature has normal distribution of the parameters such as for cloudless sky. At the cloud edges, the temperature changes fluently from low temperature for cloudless sky to the temperature of the cloud inside. 


$$
T(x)=T_{L}+\left(T_{H}-T_{L}\right) \cdot \frac{1}{1+\exp (-b x)}
$$

Figure 2 presents the course of the above function and definitions of its basic parameters. It was assumed that in the developed model, the following changeable parameters will be given: $T_{H}, T_{L}$ and $w$. $T_{L}$ parameter determines the temperature of the cloud surroundings and $T_{H}$ - the temperature of the cloud inside. The parameter $w$ defines the character of cloud temperature changes at its edge. However, $w$ does not influence directly Eq. (1). Because the function described with Eq. (1) is symmetrical to the axis of ordinates

$$
x_{T}=\frac{w}{2} \text {. }
$$

Transforming Eq. (1), in relation to $b$ parameter, and putting Eq. (2) into it, we have:

$$
b=-\frac{2}{w} \cdot \ln \left[\frac{T_{H}-T_{L}}{T(2 / w)-T_{L}}-1\right] \text {. }
$$

A value of the function determined by Eq. (1) in the point $x_{T}$ can be described as:

$$
T\left(x_{T}\right)=T_{L}+k_{T} \cdot\left(T_{H}-T_{L}\right) \text {, }
$$

where the parameter $k_{T} \in\left(\frac{1}{2}, 1\right)$ it is the coefficient determining in what degree the function of Eq. (1) has achieved the value $T_{H}$. Transforming Eq. (4), with respect to $k_{T}$ and putting it into Eq. (3) we obtain:

$$
b=-\frac{2}{w} \cdot \ln \left[\frac{1}{k_{T}}-1\right] \text {. }
$$

On the basis of the carried out analyses of real thermal images [7, 9], it was taken that $k_{T}$ parameter can be equal to 0.9 . For such a value of the parameter $k_{T}$, dependence between the parameter $b$ and the parameter $w$ can be described as:

$$
b=\frac{4,394}{w} \text {. }
$$

The case of broken sky can be assumed as some number of single clouds. Thus, their simulation is very similar to the case of single clouds.

It results from the sky thermal images that thermal images of clouds have characteristics of random distributions. A shape of clouds and their position in the space observed by a camera have to be analyzed as an atmospheric phenomenon of a local character. Thus, also these parameters have random character. Thus, for simulation, these phenomena can be described by means of some probability density function. Moreover, it results from observations of clouds formation and air movement in the clouds that this movement is, first of all, of convection character, i.e., it is vertical movement. Due to vertical movement of particles, 3D form of probability density function can be substituted by $2 \mathrm{D}$ one.

For the clouds simulation, it is necessary to apply a pseudorandom function which makes possible to generate the clouds very close to the natural ones. A pseudorandom function is in mathematical libraries of majority program languages. However, they are not adequate for the clouds generation. The main reason is that these functions are discontinuous ones. The problem is to obtain a pseudorandom function, the values of which changes "smoothly". A series of pseudorandom numbers should be generated and they should be "stroked" between subsequent values by means of an interpolation function with supplementing the values between them.

The well known practical solution of the above problem has been developed by Ken Perlin who for the first time used the noise of limited change as a rendering method [4]. The noise of the limited change is limited to some frequency range which can be understood as the maximum change between the samples. In our case, it means that the possibility exists of generation of random numbers with the maximum change between them what results in "smooth" interpolation of random samples. To generate Perlin noise, fractal summation is applied which consists in summation of the scaled harmonics of the basic noise function $n$. From such an operation, a fractal of Brown movement is formed. The basic function of the noise $n$ is a random function of uniform distribution, average value equal to zero, and the determined maximum amplitude. The Perlin noise being a fractal sum presents the equation [4]:

$$
n_{F}(x)=\sum_{i=0}^{K} \frac{n\left(\beta^{i} x\right)}{\alpha^{i}},
$$

where $K$ takes typically the value between 6 and 10. The parameter $\alpha$ checks how much irregular will be the generated noise. Low value of the parameter $\alpha$, e.g., equal to 1 gives very irregular function but a high value of this parameter gives regular ("smooth") function. In practical solutions, it happens that the terms $\beta^{i}$ and $\alpha$ are substituted by the arbitral values chosen for each $i$. The basic harmonic function is expressed by $n\left(\beta^{k} x\right)$ where $\beta$ is some positive parameter higher than unity. It was assumed in a lot of practical applications that both 
parameters $\alpha$ and $\beta$ take the value equals 2. Then, the Perlin noise is a sum of several, so-called, noise octaves, and each of subsequent octave is formed by twice increase in amplitude and twice decrease in frequency.

Figure 3 shows exemplary 2D noise determined as a sum of four the first harmonics.

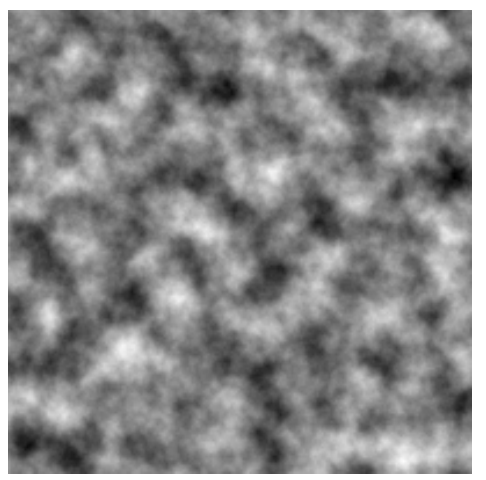

Fig. 3. Exemplary $2 D$ noise determined as a sum of the first four harmonics

The noise obtained, in the above described manner, should be transformed into clouds. To do it, the noise was used as an exponential function input, obtaining sharp "condensation level" above which the clouds are visible. The simplest and fastest way of clouds obtainment is to cut a determined value from the noise distribution and the cut of the result from bottom up to the value 0 . In this way we obtain single clouds visible in the "clean" sky. Unfortunately, we loose a part of a dynamic range for clouds. Thus, generation by means of a subtraction process can be used only in some particular situation, e.g., for generation of single clouds that are present in background of cloudless sky (having a very low temperature). Application of the algorithm of clouds generation using a subtraction method is possible because according to the conclusions from the carried out experimental results $[7,9]$, the temperature registered by a thermal camera for cloudless sky is usually below the measuring range of the camera. So, the simulation consists in the giving to cloudless sky, the universe temperature, i.e., the temperature close to zero.

\section{Generation of virtual sky in IR range}

On the basis of the described theoretical considerations, the system for simulation and sky analysis in IR range has been developed. Fundamental purpose of operation of the system for simulation and analysis of sky in $\mathrm{IR}$ range is to generate the temperatures distribution of the sky of determined parameters. The generated thermal distribution of the sky with clouds is a basis for determination of thermal images of the objects on a background of virtual sky by means of adequate IR Analysis software [5][6][8].

To generate virtual images of the sky for IR range, the program IR Sky has been developed (Fig. 4). Software can be used for simulation of sky and clouds on the basis of the parameters determined by the user, such as a range of clouds temperature and their geometrical shape. For the determined parameters, the software generates the sky thermal image. In the system for simulation and thermal analysis of the sky, a series of the advanced numerical procedures were implemented as well as algorithms of analysis and data generation for determination of indispensable characteristics and parameters. It makes possible to relatively easy accommodate the software for determination of thermal images of the sky for various environment conditions.

The basic task, realized by IR Sky program, is to generate a thermovision image of the sky on the basis of the introduced data. The software realizes generation of a thermal image of the sky and determination of thermal parameters on the basis of the input data which can be divided into the following groups of parameters: parameters describing pseudorandom generator, thermal parameters such as, e.g., a range of generated temperatures, environment parameters, geometrical parameters of the generated clouds. Moreover, using IR Sky software, thermal parameters of the obtained thermograpic image can be determined. 


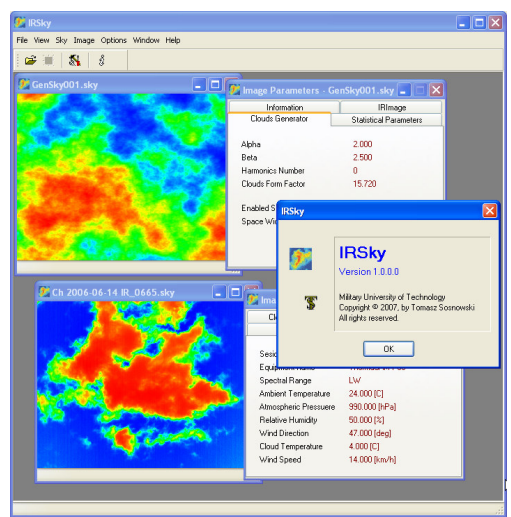

Fig. 4. A view of exemplary windows of IR Sky program for sky image generation in IR range

IR Sky software in two ways describes the sky temperature distribution. The first way is application of the registered images of the sky and, on their basis, generation of a sky image for the simulation program $I R$ Analysis. An example of the generated in this way a virtual image of the sky with clouds is shown in Fig. 5(a). The second way to obtain the distribution of clouds and sky temperatures is activation of a clouds generator, according to the parameters determined in the software. An example of a virtual image of the sky with clouds, generated by means of clouds generator, is shown in Fig. 5(b).
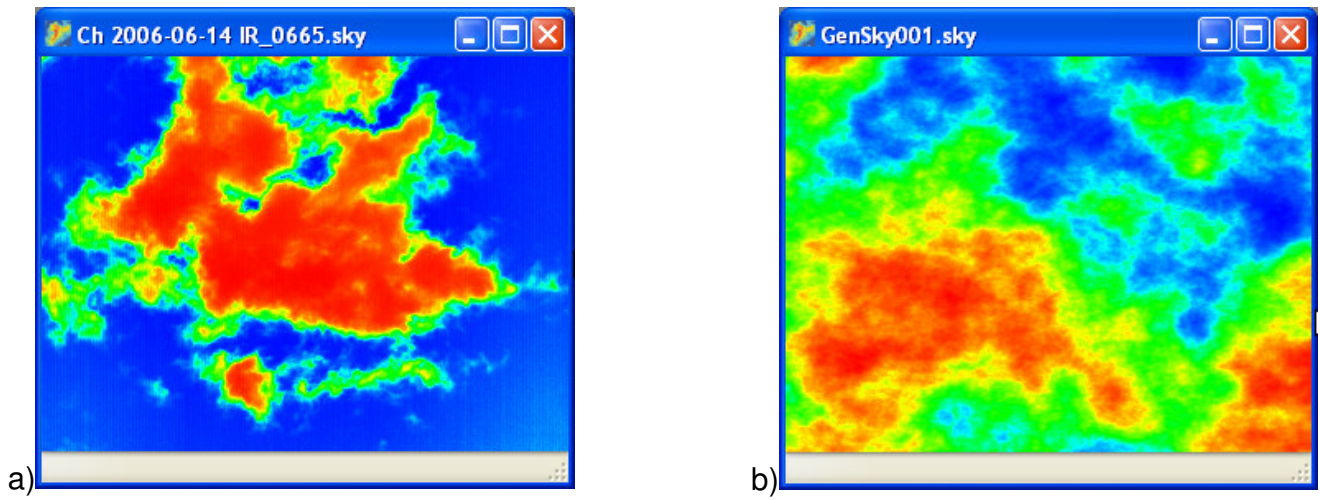

Fig. 5. A view of windows of IR Sky program containing exemplary images of the sky with clouds generated: (a) on the basis of the registered termogram and (b) generated by means of clouds generator (b).

In case of application of a clouds generator, one of key problems is proper setting the generator parameters. Principles of selection of $\alpha$ and $\beta$ parameters were described in the part describing the way of generation of the Perlin noise used in clouds generator. Essential parameter influencing the way of clouds generation is a number of the summed noise harmonics. A value of this parameter affects a shape of the generated clouds and a degree of its similarity to the clouds occurring in nature. It results from the obtained results that the number of summed components of noise harmonics has significant influence for the values lower than 8 . In case of summation of more than 8 harmonic components of noise, no changes in virtual cloud image are observed.

Each generated distribution of sky temperatures can be written as a computer file (in SKY format). In a file, despite the temperatures distribution the information is also written on the data and time of its formation, dimension of the temperature data, description introduced by the user, information on measuring conditions during the thermal image registration on the basis of which the temperature distribution was generated the temperature distribution of virtual sky, parameters of clouds generator and statistical information on virtual sky image contained in a file.

\section{Verification of modeling}

Correctness of modeling of thermal signatures can be verified by comparison of real thermal images, registered by means of a thermodetection device, with the images obtained in a computer simulation process.

Below, the verification results are shown on an exemplary thermal image. During elaboration of the investigation results, similar analyses were performed for dozens of thermal images representing various measuring conditions but having analogous results.

To verify the correctness of sky images modeling, the thermal image registered during experimental measuring sessions, by means of a thermal camera, and the computer-generated thermal image, using IR Sky program, were used. Figure 6 presents both thermal images. On the left side, the image registered with a thermal 
camera is placed and on the right side there is, corresponding with it, the image generated by means of simulation software.

Figure 7 illustrates probability density distributions of temperature distribution for thermal images of the sky with clouds. On the left, for the thermal image registered by means of a thermal camera and, on the right, for the computer-generated thermal image using IR Sky program.

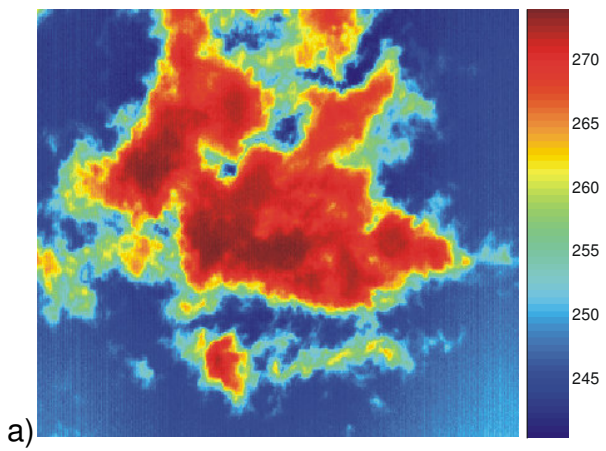

b)

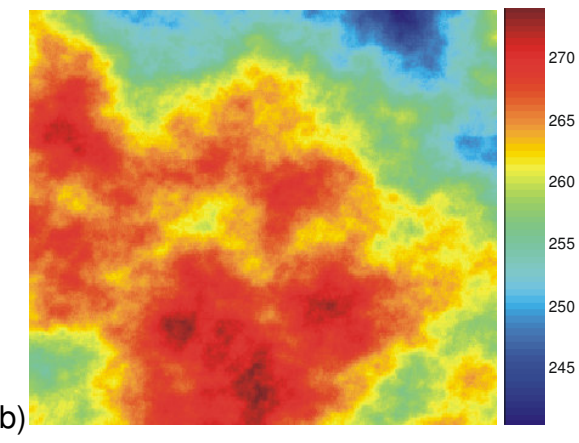

Fig. 6. Comparison of: (a) registered thermal image of the sky with clouds and (b) computer-generated thermal image using IR Sky program
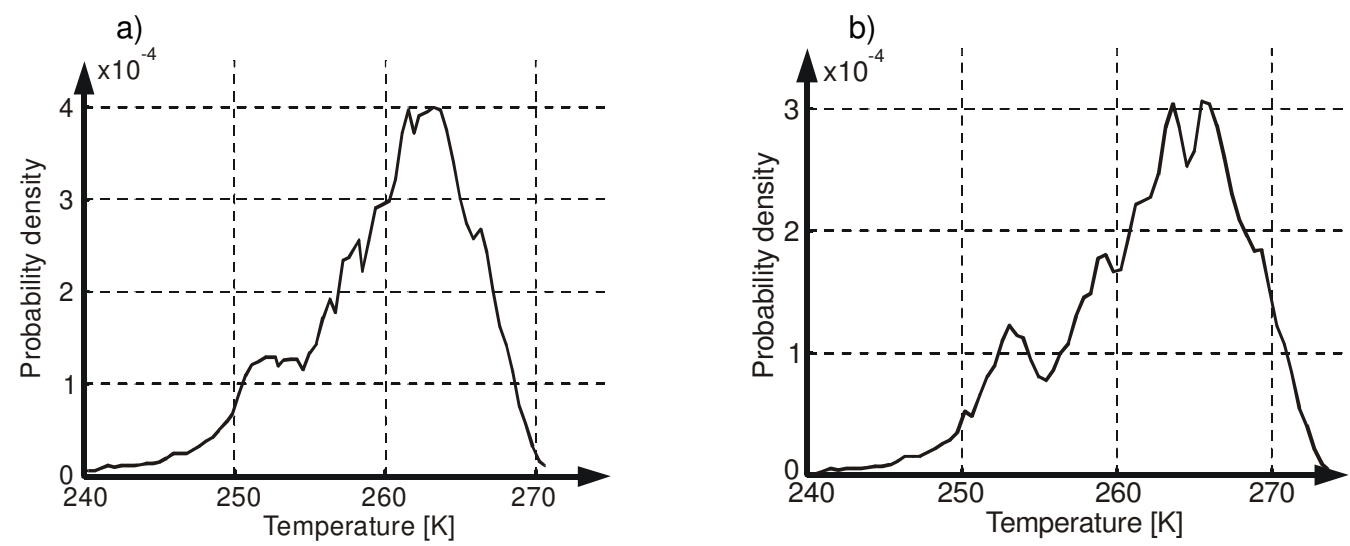

Fig. 7. Comparison of temperature probability density for: (a) registered thermal image of the sky with clouds and (b) computer-generated thermal image using IR Sky program

Diagrams of probability density of temperature distribution are close to each other. This fact testifies to the achievement of the intended purpose. Temperature distribution on the virtual thermal image properly reflects actual thermographic registration. Table 1 contains calculated statistical parameters for thermal image of Fig. 6 .

Table. 1. Statistical parameters for thermal images of the sky with clouds.

\begin{tabular}{|c|c|c|}
\hline Parameter & $\begin{array}{c}\text { Value for real } \\
\text { thermal image }[\mathrm{K}]\end{array}$ & $\begin{array}{c}\text { Value for generated } \\
\text { thermal image }[\mathrm{K}]\end{array}$ \\
\hline Minimum value & 240.113 & 240.113 \\
\hline Maxiumum value & 274.179 & 274.179 \\
\hline Average value & 260.122 & 264.299 \\
\hline Standard deviation & 10.150 & 9.841 \\
\hline
\end{tabular}

It results from thermal images analysis and the data listed in Table 1 that the difference of average temperature values on thermal images, the same an error of determination of temperature distribution in a simulation process for this case does not exceed $3 \%$.

\section{Conclusions}

Within the frame of the research works, original simulation software has been developed, providing generation of thermal images of 3D virtual objects on a determined virtual thermal background. The object and background are observed by a virtual thermodetection imaging device of the given parameters. The generation process proceeds for specified, any observation conditions of a thermal scene.

Simulation software generats thermal images of selected objects on the sky background, simulating thermographic registrations by means of short wavelength and long wavelength thermal cameras. However, a 
module construction of this software ensures its easy adaptation to generation of thermal signatures of other objects and background and makes possible to consider other parameters and phenomena during simulation. The carried out comparative analysis of the thermal images registered with a real thermal camera in field conditions and, corresponding to these registrations, the thermal images generated by means of the simulation software has confirmed the correctness of the taken assumptions and usefulness of the developed software as effective and cheap tool for obtainment of the data needed for definition of thermal signatures of real objects. Using this software, we can not only obtain reliable thermal signatures with no necessity to make expensive and time-consuming experimental investigations, but also we can predict a view of a thermal image of the scene with the investigated object in the conditions, the receiving of which during experimental investigations would be very difficult or even impossible. Due to the above mentioned virtues of the software, the detailed analysis of IR emitted, e.g., from helicopters in typical weather conditions is possible with consideration of cloud cover of the sky. Thus, the simulation software delivers indispensable data for development of effective decision algorithms for detection and recognition systems operating in IR range.

\section{REFERENCES}

[1] Genkova I. Chuck Long, Besnard T. Gillotay D., Assesing Cloud Spatial and Vertical Distribution with Infrared Cloud Analyzer, 14th ICCP, Bologna, 2004.

[2] Harris M. J.: Real-time Cloud Simulation and Rendering. Technical Report, Department of Computer Science, University of North Carolina 2003.

[3] Dobashi Y., Keneda K., Yamashita H., Okita T., Nishita T.: A simple, efficient method for realistic animation of clouds. SIGGRAPH 2000.

[4] Perlin K.: An Image Synthesizer, Computer Graphics, 1985, 19 (3), pp 287296.

[5] Sosnowski T., Dulski R., Piątkowski T., Dąbrowski M.: Programowa symulacja działania kamery

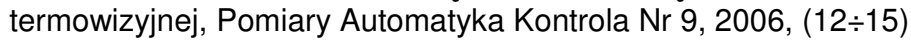

[6] Dulski R., Piątkowski T., Sosnowski T.: Metoda komputerowej symulacji sceny termalnej, Pomiary Automatyka Kontrola Nr 9, 2006, $(9 \div 11)$

[7] H. Polakowski, R. Dulski, Badanie charakterystyk radiacyjnych nieba w zakresie podczerwieni, VII Krajowa Konferencja Termografia i Termometria w Poczerwieni, Ustroń-Jaszowiec (16-18 listopad 2006).

[8] R. Dulski, H. Madura, T. Piątkowski, T. Sosnowski, Analysis of a thermal scene using computer simulations, Infrared Physics \& Technology, 49 (2007) p. 257-260.

[9] H. Polakowski, R. Dulski, K. Firmanty, Evaluation of sky and clouds IR radiation, Advanced Infrared Technology and Applications AITA 9, Leon (8-12 październik 2007).

[10] Madura, H., "Method of signal processing in passive infrared detectors for security systems", WIT Transactions on Modelling and Simulation 46, pp. 757-768, 2007.

[11] H. Madura, M. Kołodziejczyk: "Influence of sun radiation on results of non-contact temperature measurements in far infrared range", Opto-electronics Review 13, pp. 253-257, 2005.

[12] R. Dulski, H. Madura, T. Niedziela, Z. Sikorski: "Theoretical model of thermodetection system", Optica Applicata 30, pp. 443-453, 2000.

[13] Dulski R., Metoda wyznaczania odwzorowania termalnego obiektów trójwymiarowych i jej weryfikacja eksperymentalna, Rozprawa doktorska, BG WAT Warszawa (26 listopad 1998).

[14] Dulski R., Niedziela T., Verification of the correctness of thermal imaging modelling, Optica Applicata, Vol. XXXI, No 1, 2001, str. 193-202.

[15] Madura H., Modelowanie i testowanie urządzeń detekcyjnych podczerwieni, WAT 2540/98, 1998. 
http://dx.doi.org/10.21611/qirt.2008.09_05_06 